\title{
Influência do trauma cirúrgico no comportamento dos níveis séricos de albumina após cirurgia da coluna vertebral
} Influence of surgical trauma on the behavior of serum albumin after spine surgery

\author{
Influencia del trauma quirúrgico sobre el comportamiento de los \\ niveles de albúmina sérica después de cirugía de columna vertebral
}

\author{
Rafael Paiva Luciano' \\ Marcio Squassoni Leite' \\ Délio Eulálio Martins ${ }^{2}$ \\ Marcelo Wajchenberg ${ }^{3}$ \\ Eduardo Barros Puertas ${ }^{4}$
}

\section{RESUMO}

Objetivo: avaliar a variação da concentração da albumina nas cirurgias de coluna vertebral e relacioná-la às repercussões clínicas no pós-operatório. Métodos: estudo prospectivo observacional sobre os valores do nível sérico da albumina de 55 pacientes submetidos à cirurgia na coluna vertebral. As amostras de sangue foram coletadas um dia antes da cirurgia e 24 horas após o procedimento. Foi aplicado o teste $t$ pareado na avaliação da variação (pré versus pós) nos parâmetros albumina, hemoglobina e hematócrito. A presença de associação entre grau de variação nos níveis de albumina $(<1 \mathrm{~g} /$ $\mathrm{dL}$ ou $\geq 1 \mathrm{~g} / \mathrm{dL}$ ) e as variáveis de interesse: presença de complicações, necessidade de transfusão, procedimento realizado, idade e gênero do paciente e diagnóstico foram avaliadas pelo teste do $\chi^{2}$ de Pearson ou teste do $\chi^{2}$ da razão de verossimilhança quando apropriado. Resultados: a redução média

\section{ABSTRACT}

Objective: to evaluate the variation of serum albumin concentration in surgery of the spine and its relation to clinical effects in the postoperative. Methods: prospective observational study on the values of serum albumin of 55 patients undergoing spine surgery. Blood samples were collected one day before and 24 hours after the procedure. The paired t test was performed to evaluate the change parameters albumin, hemoglobin and hematocrit. The presence of association between the degree of variation in levels of albumin $(<1 \mathrm{~g} /$ $d L$ or $\geq 1 \mathrm{~g} / \mathrm{dL}$ ) and the variables of interest: complications, transfusions, procedures performed, patients' age and sex and diagnosis were assessed by $\chi^{2}$ test or the $\chi^{2}$ likelihood ratio when appropriate. Results: the average reduction of this protein was of $0.58 \mathrm{~g} / \mathrm{dL}$, ranging from 0 to 1.8 $\mathrm{g} / \mathrm{dL}$. Of the patients, $78.2 \%$ showed
\end{abstract}

\section{RESUMEN}

Objetivo: evaluar la variación de la concentración de albúmina en las cirugías de la columna vertebral y relacionarla con los efectos clínicos en el periodo postoperatorio. Métodos: estudio observacional prospectivo sobre los valores de los niveles de la albúmina de suero de 55 pacientes sometidos a cirugía de la columna vertebral. Muestras de sangre fueron tomadas un dia antes de la cirugía y 24 horas después del procedimiento. Se aplicó la prueba de t para muestras apareadas para evaluar el cambio (pre versus post) en los parámetros albúmina, la hemoglobina y el hematocrito. La presencia de asociación entre el grado de variación en los niveles de albúmina $(<1 \mathrm{~g} / \mathrm{dL}$ o $\geq 1 \mathrm{~g} / \mathrm{dL}$ ) y las variables de interés: la presencia de complicaciones, la necesidad de transfusión, el procedimiento realizado, la edad y el sexo del paciente $y$ el diagnóstico fueron evaluados por el test del $\chi^{2}$ de Pearson o el cociente

\footnotetext{
Departamento de Ortopedia e Traumatologia da Escola Paulista de Medicina da Universidade Federal de São Paulo - UNIFESP - São Paulo (SP), Brasil.

'Médico Residente do Grupo da Coluna da Disciplina de Ortopedia do Departamento de Ortopedia e Traumatologia da Escola Paulista de Medicina da Universidade Federal de São Paulo - UNIFESP - São Paulo (SP), Brasil.

${ }^{2}$ Mestre e Médico Assistente do Grupo da Coluna da Disciplina de Ortopedia do Departamento de Ortopedia e Traumatologia em Ortopedia e Traumatologia da Escola Paulista de Medicina da Universidade Federal de São Paulo - UNIFESP - São Paulo (SP), Brasil.

${ }^{3}$ Doutor e Médico Assistente do Grupo da Coluna da Disciplina de Ortopedia do Departamento de Ortopedia e Traumatologia da da Escola Paulista de Medicina da Universidade Federal de São Paulo - UNIFESP - São Paulo (SP), Brasil.

4 livre docente e Professor Associado da Disciplina de Ortopedia do Departamento de Ortopedia e Traumatologia da Escola Paulista de Medicina da Universidade Federal de São Paulo - UNIFESP - São Paulo (SP), Brasil.
} 
dessa proteína foi de $0,58 \mathrm{~g} / \mathrm{dL}$, variando de 0 a $1,8 \mathrm{~g} / \mathrm{dL}$. Dos pacientes, $78,2 \%$ apresentaram valores da albumina no período pós-operatório abaixo da faixa considerada normal. Somente dois pacientes da amostra apresentaram complicações durante os 30 dias de seguimento. O grupo com correção de deformidades apresentou $100 \%$ de pacientes com variação $\geq 1 \mathrm{~g} / \mathrm{dL}$. Conclusão: houve influência do trauma cirúrgico na redução sérica da albumina, em média, de 0,58 g/dL nas cirurgias da coluna vertebral. Não foi possível associar essa queda às complicações no período pós-operatório após seguimento de 30 dias desses pacientes. albumin values in the postoperative period below the normal range. Only two patients presented with complications during the 30 days of follow-up. The group with the correction of deformities showed $100 \%$ of patients with variation of $\geq 1$ g/dL. Conclusion: there was an influence of the surgical trauma in reducing serum albumin, on average, $0.58 \mathrm{~g} / \mathrm{dL}$ in surgery of the spine. It was not possible to associate this decrease to complications in the postoperative period after 30 days of follow-up of these patients. de probabilidad $\chi^{2}$, cuando necesario. Resultados: la reducción media de esta proteina fue de $0,58 \mathrm{~g} / \mathrm{dL}$, que varía de 0 a 1,8 g/dL. De los pacientes, 78,2\% tuvieron valores de albúmina en el postoperatorio por debajo del rango normal. Sólo dos pacientes presentaron complicaciones durante los 30 días de seguimiento. El grupo con la corrección de las deformidades presentó el $100 \%$ de pacientes con mayor o $\geq 1 \mathrm{~g} /$ dL. Conclusión: hubo una influencia del trauma quirúrgico en la reducción de la albúmina sérica, en promedio, de $0,58 \mathrm{~g} / \mathrm{dL}$ en las cirugías de la columna vertebral. No se puede asociar esta caída a las complicaciones en el postoperatorio después de 30 días de seguimiento de esos pacientes.

\section{DESCRIPTORES: Albúmina sérica;
Procedimientos quirúrgicos
operativos; Columna vertebral;
Enfermedades de la columna
vertebral; Columna vertebral/ \\ DESCRIPTORES: Albúmina sérica;
Procedimientos quirúrgicos
operativos; Columna vertebral;
Enfermedades de la columna
vertebral; Columna vertebral/ \\ DESCRIPTORES: Albúmina sérica;
Procedimientos quirúrgicos
operativos; Columna vertebral;
Enfermedades de la columna
vertebral; Columna vertebral/ \\ DESCRIPTORES: Albúmina sérica;
Procedimientos quirúrgicos
operativos; Columna vertebral;
Enfermedades de la columna
vertebral; Columna vertebral/ \\ DESCRIPTORES: Albúmina sérica;
Procedimientos quirúrgicos
operativos; Columna vertebral;
Enfermedades de la columna
vertebral; Columna vertebral/ cirugía

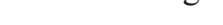

DESCRITORES: Albumina sérica; Procedimentos cirúrgicos operatórios; Coluna vertebral; Doenças da Coluna Vertebral; Coluna vertebral/cirurgia
KEYWORDS: Serum albumin; Surgical procedures, operative; Spine; Spinal diseases; Spine/surgery

\section{INTRODUÇÃO}

A albumina é a proteína plasmática presente em maior quantidade no sangue, totalizando cerca de $60 \%$ destas. É sintetizada no fígado e secretada na corrente sanguínea como um produto de 585 aminoácidos de cadeia única, dispostos em uma estrutura terciária ${ }^{1,2}$. Está distribuída em dois compartimentos: $60 \%$ no espaço extravascular e o restante circulante na corrente sanguínea. Esse equilíbrio entre plasma e espaço intersticial é mantido pelo constante transporte transcapilar da albumina em direção ao "terceiro espaço", também chamado de espaço intersticial ${ }^{3}$.

A principal função dessa proteína plasmática é a manutenção da pressão coloidosmótica, sendo responsável por cerca de $80 \%$ da pressão intravascular ${ }^{3}$. Na corrente sanguínea, também é fundamental no tamponamento ácido/base, mantendo o $\mathrm{pH}$ necessário às reações enzimáticas vitais para a homeostasia ${ }^{1}$.

A mensuração da albumina plasmática é considerada um método simples e de baixo custo para a avaliação nutricional de pacientes cirúrgicos. $\mathrm{O}$ valor considerado normal da concentração de albumina no sangue é de 3,5 a 5,5 $\mathrm{g} / \mathrm{dL}^{4,5}$. Em níveis baixos, essa proteína perde sua capacidade fisiológica, e a diminuição afeta a difusão vascular de nutrientes, com repercussão nos sistemas de defesa e na manutenção do equilíbrio do organismo.
A concentração sérica de albumina, além de ser um parâmetro clássico de avaliação nutricional, sofre variações importantes em resposta ao estresse metabólico ou à lesão tecidual. Por isso, é considerada uma proteína negativa da "fase aguda", ou seja, aquela que tem sua produção diminuída em reposta ao estímulo inflamatório ${ }^{4,6,7}$. A lesão traumática e os procedimentos cirúrgicos são frequentemente seguidos por edema tecidual e baixa concentração de proteína plasmática, indicando um extravasamento da albumina para o espaço intersticial ${ }^{8}$.

Alguns estudos têm associado baixos níveis da albumina sérica à piora do prognóstico pós-operatório ${ }^{6,9}$. Complicações como infecção profunda ou superficial, pneumonia, sepse e aumento da mortalidade no período pós-operatório também já foram relacionadas à baixa concentração plasmática de albumina ${ }^{5,6,9,10}$.

Apesar de estar bem estabelecido que a hipoalbuminemia está associada ao aumento do risco de morbimortalidade no período pós-cirúrgico, essa avaliação é, muitas vezes, menosprezada pelos cirurgiões. Em relação à cirurgia da coluna vertebral, a variação da albumina sérica após o procedimento cirúrgico, bem como sua repercussão clínica, foram pouco discutidas na literatura. Desse modo, o objetivo deste estudo é avaliar a variação da concentração da albumina nas cirurgias de coluna vertebral e relacionála às repercussões clínicas no pós-operatório. 


\section{MÉTODOS}

Foi realizado estudo clínico, prospectivo e observacional sobre valores pré e pós-cirúrgicos do nível sérico da albumina de 55 pacientes submetidos à cirurgia na coluna vertebral, durante o período de Junho a Dezembro de 2009, após aprovação do Comitê de Ética e Pesquisa (CEP $n^{\circ}$ 0898/09) e após assinatura do Termo de Consentimento Livre Esclarecido. Os procedimentos realizados foram: discectomia, com e sem artrodese; artrodese de um ou mais níveis, com ou sem descompressão; e correção de deformidades como escoliose e doença de Scheuermann. Foram incluídas neste estudo tanto as cirurgias de urgência como as realizadas de maneira eletiva nos três segmentos da coluna vertebral.

Como critério de inclusão, os pacientes deveriam ter 15 anos completos e indicação cirúrgica para tratamento das seguintes doenças da coluna vertebral: hérnia de disco, fratura vertebral, estenose lombar, espondilolistese, escoliose e doença de Scheuermann. Foram excluídos deste estudo os pacientes que apresentavam doenças sistêmicas que alteram o metabolismo fisiológico das proteínas, como as glomerulonefrites, doenças disabsortivas, hepatopatias e coagulopatias.

As amostras do sangue foram coletadas um dia antes da cirurgia e 24 horas após o procedimento. Além da análise da albumina, também foram dosados a hemoglobina e o hematócrito. O sangue colhido foi submetido ao analisador bioquímico Olympus AU640, pelo método colorimétrico verde de bromocresol, com valor de referência da albumina sérica entre 3,5 e $5,5 \mathrm{~g} / \mathrm{dL}$. O tempo cirúrgico e a quantidade de hemoconcentrado de hemácias transfundidas ao paciente foram outras variáveis incluídas neste trabalho.

Os pacientes foram também avaliados quanto às complicações clínicas precoces e condições de ferida operatória, com seguimento de 30 dias após o procedimento cirúrgico.

As variáveis qualitativas foram representadas por frequência absoluta (n) e relativa (\%), e as quantitativas por média, desvio-padrão (DP), mediana, valores mínimo e máximo. O teste de Kolmogorov-Smirnov foi aplicado para testar a presença de distribuição normal nos parâmetros quantitativos. Foi aplicado o teste $t$ pareado na avaliação da variação (pré versus pós) nos parâmetros albumina, hemoglobina e hematócrito. A presença de associação entre grau de variação nos níveis de albumina $(<1$ ou $\geq 1 \mathrm{~g} / \mathrm{dL})$ e as variáveis de interesse: presença de complicações, necessidade de transfusão, procedimento realizado, idade do paciente, sexo do paciente e diagnóstico foram avaliadas pelo teste do $\chi^{2}$ de Pearson ou teste do $\chi^{2}$ da razão de verossimilhança quando apropriado. Foi adotado o nível de significância de $0,05(\alpha=5 \%)$, e níveis descritivos (p) inferiores a esse valor foram considerados significantes e representados por *. Todas as análises foram feitas pelo software SPSS for Windows versão 15.0 .

\section{RESULTADOS}

A idade dos pacientes variou de 15 a 80 anos, com uma média de 43,2 anos, e apenas $18 \%$ tinham mais que 60 anos. Cerca de $50 \%$ eram do gênero masculino, e o diagnóstico mais encontrado foi hérnia discal lombar $(56,4 \%)$. Para esse diagnóstico realizou-se discectomia simples em 21 pacientes. A duração média de todos os procedimentos foi de 163,7 minutos, variando de 50 a 720 minutos (Tabela 1).

\section{TABELA 1 - Características da amostra de pacientes}

\begin{tabular}{lc}
\hline Variáveis & $(\mathbf{n}=55)$ \\
\hline ldade (anos) & $43,2(16,7)$ \\
Média (DP) & 38 \\
Mediana & $15-80$ \\
Mínimo - máximo & \\
ldade - n (\%) & $45(81,8)$ \\
Até 59 anos & $10(18,2)$ \\
60 anos ou mais & \\
Sexo - n (\%) & $27(49,1)$ \\
Masculino & $28(50,9)$ \\
Feminino & \\
Diagnóstico - n (\%) & $4(7,3)$ \\
Deformidades & $6(10,9)$ \\
Estenose lombar & $31(56,4)$ \\
Hérnia lombar & $3(5,5)$ \\
Espondilolistese & $11(20,0)$ \\
Fraturas & \\
Procedimento - $n$ (\%) & $3(5,5)$ \\
Correção de deformidades & $9(16,4)$ \\
Descompressão + Artrodese 1 nível & $11(20,0)$ \\
Descompressão + Artrodese 2 níveis & $6(10,9)$ \\
Descompressão + Artrodese $>2$ níveis & $21(38,2)$ \\
Discectomia simples & $5(9,1)$ \\
Artrodese cervical & \\
Tempo de cirurgia (minutos) & $163,7(133,8)$ \\
Média (DP) & 130 \\
Mediana & $50-720$ \\
Mínimo - máximo & \\
\hline
\end{tabular}

A concentração do valor sérico da albumina reduziu de 3,52 para $2,94 \mathrm{~g} / \mathrm{dL}$ no período pós-operatório $(\mathrm{p}<0,001)$, com uma variação média de $0,58 \mathrm{~g} / \mathrm{dL}$ e redução máxima de $1,8 \mathrm{~g} / \mathrm{dL}$. Em sete pacientes, a diminuição da albumina foi $\geq 1 \mathrm{~g} / \mathrm{dL}(12,7 \%)$ : três pacientes após correção de deformidade; dois após descompressão e artrodese de dois níveis; um após descompressão e artrodese de um nível; e um após discectomia simples. Dos pacientes, $78,2 \%$ apresentaram valores da albumina no período pós-operatório abaixo da faixa considerada normal. Essa porcentagem dobrou em relação à avaliação pré-operatória, em que $38,2 \%$ da amostra tinha valores $<3,5 \mathrm{~g} / \mathrm{dL}$ (Tabela 2). 
TABELA 2 - Níveis de Albumina dos pacientes ao longo do seguimento

\begin{tabular}{lccc}
\hline $\begin{array}{l}\text { Albumina (g/dL) } \\
\text { (n=55) }\end{array}$ & $\begin{array}{c}\text { Avaliação } \\
\text { pré }\end{array}$ & $\begin{array}{c}\text { Avaliação } \\
\text { pós }\end{array}$ & $\begin{array}{c}\text { Variação } \\
\text { (pré - pós) }\end{array}$ \\
\hline Média (DP) & $3,52(0,57)$ & $2,94(0,55)$ & $0,58(0,40)$ \\
Mediana & 3,6 & 3,0 & 0,6 \\
Mínimo / máximo & $1,9 / 4,7$ & $1,1 / 4,0$ & $0,0 / 1,8$ \\
Préx Pós & & & $p<0,001$ * \\
Niveis da albumina & & & \\
$\geq 3,5 \mathrm{~g} / \mathrm{dL}$ & $34(61,8)$ & $12(21,8)$ & \\
$2,2 \mathrm{a} 3,4 \mathrm{~g} / \mathrm{dL}$ & $20(36,4)$ & $40(72,7)$ & \\
$\leq 2,1 \mathrm{~g} / \mathrm{dL}$ & $1(1,8)$ & $3(5,5)$ & \\
Variação da albumina & & & \\
$<1 \mathrm{~g} / \mathrm{dL}$ & & & $48(87,3)$ \\
$\geq 1 \mathrm{~g} / \mathrm{dL}$ & & & $7(12,7)$ \\
\hline
\end{tabular}

Foram observadas variações estatisticamente significativas entre as avaliações pré e pós de hemoglobina $(\mathrm{p}<0,001)$ e de hematócrito $(p<0,001)$. Nos valores da hemoglobina, a redução média observada foi de $2,1 \mathrm{~g} / \mathrm{dL}(\mathrm{DP}=1,5 \mathrm{~g} / \mathrm{dL})$, reduzindo de uma média de $13,5 \mathrm{~g} / \mathrm{dL}(\mathrm{DP}=1,6)$ para 11,4 $\mathrm{g} / \mathrm{dL}(\mathrm{DP}=1,8)$. Foi necessária a transfusão de concentrado de hemácias em seis pacientes: dois com diagnóstico de estenose lombar, um com escoliose degenerativa, um com cifose de Scheuermann, um com fratura de L2 e um com doença degenerativa da coluna lombar. Não foi encontrada associação estatisticamente significativa entre a necessidade de transfusão e a variação nos níveis de albumina $(p=0,577)$.

Somente dois pacientes da amostra apresentaram complicações durante os 30 dias de seguimento. Observou-se pneumonia em um paciente submetido à dupla via para correção de cifose e uma infecção superficial após artrodese cervical. O grupo sem complicações apresentou $11,3 \%$ dos pacientes com variação $\geq 1 \mathrm{~g} / \mathrm{dL}$ nos níveis de albumina, e no grupo com complicações essa proporção foi de $50 \%(\mathrm{p}=0,240)$.

Ao relacionarmos os dados demográficos da amostra com a variação dos níveis de albumina, observamos que $18,5 \%$ dos homens apresentaram variação $\geq 1 \mathrm{~g} / \mathrm{dL}$, e no grupo feminino essa proporção foi de $7,1 \%(p=0,252)$. Todos os pacientes que tiveram uma variação $>1 \mathrm{~g} / \mathrm{dL}$ tinham menos que 60 anos de idade, porém, sem significância estatística.

Encontrou-se associação estatisticamente significante entre os procedimentos e a variação nos níveis de albumina. O grupo com correção de deformidades apresentou $100 \%$ de pacientes com variação $\geq 1 \mathrm{~g} / \mathrm{dL}$, e nos demais grupos de procedimentos essa proporção variou entre $0 \% \mathrm{e}$ $18,2 \%(\mathrm{p}=0,004)$. O grupo com discectomia simples apresentou apenas $4,8 \%$ de pacientes com variação $\geq 1 \mathrm{~g} / \mathrm{dL}$, e no grupo com demais procedimentos essa proporção foi de $17,6 \%$, mas sem diferença significante entre os grupos.

$\mathrm{Na}$ análise da associação entre os diagnósticos e a variação nos níveis de albumina, $75 \%$ do grupo com deformidades apresentou variação $\geq 1 \mathrm{~g} / \mathrm{dL}$, e nos demais grupos de diagnósticos essa proporção variou entre $0 \%$ e $12,9 \%$. Essa análise obteve diferença estatisticamente significante $(\mathrm{p}=0,009)$.

\section{DISCUSSÃO}

A hipoalbuminemia pós-cirúrgica foi inicialmente descrita como um fator de risco para o aumento da morbimortalidade de pacientes no período pós-operatório por Rhoads e Alexander em $1955^{10}$. Desde então, estudos subsequentes têm associado o baixo nível da albumina sérica a piores prognósticos pós-operatórios. A determinação do nível sérico dessa proteína é um importante marcador da avaliação nutricional e identifica pacientes cirúrgicos com déficit nutricional que, sabidamente, têm maior risco de complicações pós-operatórias, ou até de morte, quando comparados àqueles bem nutridos que sofreram o mesmo procedimento cirúrgico $0^{6,9,11,12}$.

Associado ao fato de que a hipoalbuminemia é um fator de risco independente para o aumento da morbimortalidade após procedimentos cirúrgicos, também é notória a associação da diminuição do nível sérico da albumina durante e após os procedimentos cirúrgicos ${ }^{8,13}$. Essa hipoalbuminemia pós-cirúrgica é explicada, em parte, pelas alterações da permeabilidade vascular, que ocorrem em resposta ao estímulo inflamatório e, também, pela característica da diminuição na síntese hepática dessa proteína após estresse metabólico ${ }^{7,14,15}$. $\mathrm{Na}$ análise realizada, o tempo cirúrgico não foi determinante para a diminuição significativa da concentração sanguínea da albumina. Os procedimentos nos pacientes com variação maior que $1 \mathrm{~g} /$ dL tiveram uma duração média de 163 minutos, e naqueles em que a variação foi menor do que $1 \mathrm{~g} / \mathrm{dL}$, a média da duração foi de 155 minutos. Esse dado corrobora o fato de que o trauma cirúrgico é fator determinante para a alteração da síntese da albumina no período pós-operatório.

Neste estudo foi possível identificar a queda dos níveis séricos da albumina após cirurgias da coluna vertebral, em média, de $0,58 \mathrm{~g} / \mathrm{dL}$, com significância estatística, sendo que a maior queda atingiu a diferença de $1,8 \mathrm{~g} / \mathrm{dL}$ entre os valores pré e pós-operatórios. Este resultado chama a atenção no sentindo de alertar o cirurgião de coluna vertebral de que a queda dos níveis da albumina, tão citada nas outras especialidades cirúrgicas, também ocorre na cirurgia da coluna vertebral. No entanto, a falta de dados na literatura sobre a queda de albumina aponta o desconhecimento dos cirurgiões dessa especialidade.

A hipoalbuminemia, condição caracterizada por níveis séricos menores que 3,5 g/dL, esteve presente em 78,2\% dos pacientes deste estudo após 24 horas do procedimento cirúrgico. Essa condição clínica está associada a uma série de alterações fisiológicas que podem levar a complicações pós-operatórias e até à morte 4 . Christou et al., em 1981, demonstraram que pacientes com anergia cutânea apresentavam níveis baixos de albumina propondo que essa alteração sanguínea diminui a resposta imunológica da pele, aumentando a chance de infecções da ferida operatória ${ }^{16}$. 
Gibbs et al., em 1999, baseados em uma ampla análise dos níveis séricos da albumina e sua relação com complicações ocorridas até o $30^{\circ}$ dia de pós-operatório, concluíram que, dentre as 62 variáveis analisadas, a concentração sérica da albumina antes da cirurgia foi o dado analisado que melhor se relacionava à morbimortalidade no pós-operatório. Esses autores demonstraram, a partir do cálculo do odds ratio do nível da albumina, que a variação maior que $1 \mathrm{~g} / \mathrm{dL}$ aumenta duas vezes a chance de ocorrer a morte destes pacientes ${ }^{11}$. No estudo atual, apenas $12,7 \%$ dos pacientes apresentaram uma variação maior que $1 \mathrm{~g} /$ $\mathrm{dL}$, sendo que o procedimento em que mais ocorreu essa variação foi a correção de deformidades - que, apesar de não envolver a descompressão do canal vertebral, é uma cirurgia com maior lesão tecidual devido à extensa dissecção de partes moles, o que gera um estresse metabólico mais intenso e com maior espoliação dos pacientes.

Em 2007, na análise de 200 cirurgias do sistema digestório alto, Ryan et al. demonstraram que, em pacientes com albumina menor do que $2 \mathrm{~g} / \mathrm{dL}$, no primeiro dia pós-operatório, o índice de complicações dobrava quando comparados aos pacientes com valor maior do que $2 \mathrm{~g} / \mathrm{dL}^{12}$. Na presente amostra, 5,5\% dos pacientes apresentaram níveis pós-operatórios menores do que $2 \mathrm{~g} / \mathrm{dL}$, e em nenhum deles foram observadas complicações. Esse dado pode ser explicado pelo fato de que a variação da albumina nesses pacientes foi menor do que $1 \mathrm{~g} / \mathrm{dL}$. Gibbs et al., em 1999, por meio da análise sérica pré-operatória, observaram que havia um aumento da mortalidade de $1 \%$ para $29 \%$ quando os níveis pré-operatórios da albumina eram, respectivamente, maiores que $4,6 \mathrm{~g} / \mathrm{dL}$ e menores que $2,1 \mathrm{~g} / \mathrm{dL}^{11}$. Na amostra atual, encontramos apenas um paciente com nível pré-operatório sérico de albumina menor que $2,1 \mathrm{~g} / \mathrm{dL}$ que não apresentou complicação no seu acompanhamento.

$\mathrm{Na}$ análise das características demográficas relacionadas aos níveis séricos de albumina, os homens apresentaram maior tendência de queda dos níveis de albumina, porém, sem significância estatística. Ryan et al., em 2007, não observaram diferença entre os sexos em sua análise pós-operatória; no entanto, Rady et al., em 1997 observaram que o sexo feminino e pacientes com mais de 60 anos apresentavam valores de albumina menores em consequência do aumento do transporte da albumina para o espaço extravascular ${ }^{12,15}$. As outras variáveis estudadas, como queda da hemoglobina e hematócrito, transfusão sanguinea e idade, seguiram a tendência dos outros estudos citados e não apresentaram diferença com significância estatística.

Mesmo não havendo significância estatística na relação do número de complicações com a queda maior que $1 \mathrm{~g} /$ $\mathrm{dL}$, provavelmente devido à baixa ocorrência, verificou-se que em $50 \%$ dos pacientes que apresentaram complicações a variação da albumina foi maior que $1 \mathrm{~g} / \mathrm{dL}$. No entanto, nesta amostra só ocorreram duas complicações: pneumonia em um paciente submetido à dupla via para correção de cifose, procedimento associado a um maior número de complicações pelo maior tempo cirúrgico e, também, pela abordagem torácica; e infecção superficial em um paciente com fratura-luxação cervical, que teve um decréscimo menor que $1 \mathrm{~g} / \mathrm{dL}$. Ambas as situações tiveram boa evolução após tratamento com antibioticoterapia.

As deformidades representaram o diagnóstico mais relacionado à diminuição da albumina sanguínea após o tratamento cirúrgico. Mas, apesar da falta de dados na literatura sobre essa relação, há uma preocupação por parte dos especialistas de coluna de tentar associar uma melhora das condições nutricionais desses pacientes por meio da nutrição parenteral aos menores índices de complicações ${ }^{17,18}$. Hu et al., em 1997, concluíram que o uso da nutrição parenteral total melhorou os parâmetros nutricionais dos pacientes submetidos a cirurgias para correção da deformidade em duas etapas: via anterior e, após uma semana, via posterior ${ }^{17}$.

Os resultados apresentados neste estudo demonstram uma tendência de queda da albumina após os procedimentos da coluna vertebral e, também, um estado de hipoalbuminemia desses pacientes no pós-operatório imediato. No entanto, futuros trabalhos devem ser conduzidos no sentido de associar essa queda às repercussões clínicas nos pacientes, bem como a necessidade da reposição dessa proteína no pós-operatório das cirurgias espinhais.

\section{CONCLUSÃO}

Houve influência do trauma cirúrgico na redução sérica da albumina, em média, de $0,58 \mathrm{~g} / \mathrm{dL}$ nas cirurgias da coluna vertebral. Porém, não foi possível associar essa queda às complicações no período pós-operatório após um seguimento de 30 dias desses pacientes.

\section{REFERÊNCIAS}

1. Peters T Jr., All about albumin: biochemistry, genetics, and medical applications. San Diego, California: Academic Press; 1996.
2. Blindauer CA, Harvey I, Bunyan KE, Stewart AJ, Sleep D, Harrison DJ, et al., Structure, properties, and engineering of the major zinc binding site on human albumin. J Biol Chem. 2009. 284(34):23116-24.

3. Boldt J. Use of albumin: an update. $\mathrm{Br}$ J Anaesth. 2010;104(3):276-84.
4. Franch-Arcas G. The meaning of hypoalbuminaemia in clinical practice. Clin Nutr. 2001;20(3):265-9.

5. Thompson JS, Burrough CA, Green JL, Brown GL. Nutritional screening in surgical patients. J Am Diet Assoc. 1984;84(3):337-8. 
6. Leite HP, Fisberg M, de Carvalho WB, de Camargo Carvalho AC. Serum albumin and clinical outcome in pediatric cardiac surgery. Nutrition. 2005;21(5):553-8.

7. Alberti LR, Petroianu A, Zac RI, Andrade JC Jr. Relationship between surgical trauma and albumin concentration in the postoperative period of digestive system operations. Minerva Chir. 2008;63(5):357-62.

8. Kongstad L, Möller AD, Grände PO. Reflection coefficient for albumin and capillary fluid permeability in cat calf muscle after traumatic injury. Acta Physiol Scand. 1999;165(4):369-77.

9. Kudsk KA, Tolley EA, DeWitt RC, Janu PG, Blackwell AP, Yeary S, et al. Preoperative albumin and surgical site identify surgical risk for major postoperative complications. JPEN J Parenter Enteral Nutr. 2003;27(1):1-9.

10.Rhoads JE, Alexander CE. Nutritional problems of surgical patients. Ann N Y Acad Sci. 1955;63(2):268-75.
11.Gibbs J, Cull W, Henderson W, Daley J, Hur K, Khuri SF. Preoperative serum albumin level as a predictor of operative mortality and morbidity: results from the National VA Surgical Risk Study. Arch Surg. 1999;134(1):36-42.

12. Ryan AM, Hearty A, Prichard RS, Cunningham A, Rowley SP, Reynolds JV. Association of hypoalbuminemia on the first postoperative day and complications following esophagectomy. J Gastrointest Surg. 2007;11(10):1355-60.

13. Barle H, Hållström L, Essèn P, Thörne A, McNurlan MA, Garlick PJ, et al. The synthesis rate of albumin decreases during laparoscopic surgery. Clin Physiol Funct Imaging. 2004;24(2):91-5.

14.Ballmer PE. Causes and mechanisms of hypoalbuminaemia. Clin Nutr. 2001;20(3):271-3.

15.Rady MY, Ryan T, Starr NJ. Clinical characteristics of preoperative hypoalbuminemia predict outcome of cardiovascular surgery. JPEN J Parenter Enteral Nutr. 1997;21(2):81-90.
16.Christou NV, Meakins JL, MacLean LD. The predictive role of delayed hypersensitivity in preoperative patients. Surg Gynecol Obstet. 1981;152(3):297-301.

17.Hu SS, Fontaine F, Kelly B, Bradford DS. Nutritional depletion in staged spinal reconstructive surgery. The effect of total parenteral nutrition. Spine (Phila Pa 1976). 1998;23(12):1401-5.

18.Mullen JL, Buzby GP, Matthews DC, Smale BF, Rosato EF. Reduction of operative morbidity and mortality by combined preoperative and postoperative nutritional support. Ann Surg. 1980;192(5):604-13.

\section{Correspondência:}

$$
\begin{aligned}
& \text { Rafael Paiva Luciano } \\
& \text { Rua Borges Lagoa, } 783 \text { - 5 andar } \\
& \text { Vila Clementito } \\
& \text { CEP 04038-032 - São Paulo (SP), } \\
& \text { Brasil } \\
& \text { E-mail: rafaelluciano@ig.com.br }
\end{aligned}
$$

